\title{
A Wetting Transition of Ionic Substrate by Modulating Surface Charge
}

\section{Distribution}

Peng Jiang ${ }^{1,2}$, Hongguan $\mathrm{Wu}^{2}$, Leying Qing ${ }^{2}$, Xiaofei $\mathrm{Xu}^{2}$, Zhehui Jin ${ }^{3}$, Li Yang ${ }^{1, *}$, and Shuangliang Zhao ${ }^{2, *}$

${ }^{1}$ Key Laboratory of Green Chemical Process of Ministry of Education, Key Laboratory of Novel Reactor and Green Chemical Technology of Hubei Province, School of Chemical Engineering and Pharmacy, Wuhan Institute of Technology, Wuhan 430205, China

${ }^{2}$ State Key Laboratory of Chemical Engineering and School of Chemical Engineering, East China University of Science and Technology, Shanghai 200237, China

${ }^{3}$ School of Mining and Petroleum Engineering, Department of Civil and Environmental Engineering, University of Alberta, Edmonton AB T6G 1H9, Canada

*Corresponding authors. liyang@wit.edu.cn (LY) and szhao@ecust.edu.cn (SZ) 


\section{Simulation details}

The molecular dynamics simulation for acetonitrile near a graphene flat substrate is performed by using LAMMPS ${ }^{1}$. The simulation system contains 864 acetonitrile molecules and a singlelayer graphene sheet, and the lengths are $L_{x}=4.175 \mathrm{~nm}, L_{y}=4.254 \mathrm{~nm}$ and $L_{z}=7.5 \mathrm{~nm}$. The graphene atoms are fixed at their perfect lattice positions, while the acetonitrile molecule is held rigid through the SHAKE algorithm ${ }^{2}$. The dispersion interaction and the Coulomb interaction are both truncated at a cutoff distance of $1.2 \mathrm{~nm}$, and the parameters have been listed in the main text. The long-range Coulomb interactions are are calculated using the particleparticle particle-mesh method ${ }^{3}$. The equation of motion is integrated by the velocity Verlet algorithm ${ }^{4}$. The fluid temperature is maintained at $300 \mathrm{~K}$ via Nosé-Hoover thermostat ${ }^{5}$ with a time constant of $0.1 \mathrm{ps}$. Total simulation time is $1 \mathrm{~ns}$ with a time step of $1 \mathrm{fs}$. The first $0.5 \mathrm{~ns}$ is used to equilibrate the system and the rest is used to collect data.

\section{References:}

1. Plimpton, S., Fast Parallel Algorithms for Short-Range Molecular Dynamics. Journal of Computational Physics 1995, 117, 1-19.

2. Ryckaert, J. P.; Ciccotti, G.; Berendsen, H. J. C., Numerical integration of the Cartesian equations of motion of a system with constraints: Molecular dynamics of n-alkanes. Journal of Computational Physics 1977, 23, 327-341.

3. Hockney, R. W.; Eastwood, J. W., Computer Simulation Using Particles. Adam Hilger 1989.

4. W.C. Swope, H. C. A., P.H. Berens, K.R. Wilson, A computer simulation method for the calculation of equilibrium constants for the formation of physical clusters of molecules: Application to small water clusters. Journal of Chemical Physics 1982, 76, 637-649.

5. Nose, S., Constant-temperature molecular dynamics. Journal of Physics Condensed Matter 1990, 2, SA115. 